Article

\title{
Association Rules for Understanding Policyholder Lapses
}

\author{
Himchan Jeong, Guojun Gan (iD) and Emiliano A. Valdez * \\ Department of Mathematics, University of Connecticut, Storrs, CT 06269-1009, USA; \\ himchan.jeong@uconn.edu (H.J.); guojun.gan@uconn.edu (G.G.) \\ * Correspondence: emiliano.valdez@uconn.edu; Tel.: +1-860-486-6331
}

Received: 25 May 2018; Accepted: 04 July 2018; Published: 8 July 2018

check for updates

\begin{abstract}
For automobile insurance, it has long been implied that when a policyholder made at least one claim in the prior year, the subsequent premium is likely to increase. When this happens, the policyholder may seek to switch to another insurance company to possibly avoid paying for a higher premium. In such situations, insurers may be faced with the challenges of policyholder retention by keeping premiums low in the face of competition. In this paper, we seek to find empirical evidence of possible association between policyholder switching after a claim and the associated change in premium. In accomplishing this goal, we employ the method of association rule learning, a data mining technique that has its origins in marketing for analyzing and understanding consumer purchase behavior. We apply this unique technique in two stages. In the first stage, we identify policyholder and vehicle characteristics that affect the size of the claim and resulting change in premium regardless of policy switch. In the second stage, together with policyholder and vehicle characteristics, we identify the association among the size of the claim, the level of premium increase and policy switch. This empirical process is often challenging to insurers because they are unable to observe the new premium for those policyholders who switched. However, we used nine-year claims data for the entire Singapore automobile insurance market that allowed us to track information before and after the switch. Our results provide evidence of a strong association among the size of the claim, the level of premium increase and policy switch. We attribute this to the possible inefficiency of the insurance market because of the lack of sharing and exchange of claims history among the companies.
\end{abstract}

Keywords: data mining; association rule learning; policyholder lapse; auto insurance; market inefficiency

\section{Introduction}

In several jurisdictions, anyone who owns a motor vehicle must have auto insurance coverage at all times. At a minimum, the insurance must provide some level of liability protection, although many motor vehicle owners opt for a more comprehensive coverage that additionally provides for insurance protection against collision and vehicle damage. The automobile insurance market is very competitive where motor vehicle owners can shop freely for insurance coverage that are generally homogeneous but prices are extremely competitive. For insurance companies then, customer loyalty and policy retention become an important strategic management because it is generally more cost efficient to retain existing policies than to acquire new ones (see McClenahan (2001)).

It has long been held that when the policyholder made a claim in the prior year, the subsequent premium is likely to increase. The level of premium depends on many factors such as the frequency and severity of the claims made. In some jurisdictions, the practice of implementing a bonus-malus system allows for a well-defined mechanism of premium determination triggered by claims. In a bonus-malus 
system, premiums increase the following policy year whenever claims are made this policy year. On the other hand, discounted premiums are provided if there is no claim (see Lemaire (1985)). In Singapore, their bonus-malus system is more formally referred to as a No-Claims Discount (NCD) system. It has a baseline premium with a $0 \%$ discount and discounts are provided in increments of $10 \%$ per year for up to $50 \%$.

When claims trigger premium increase, it becomes more attractive for the policyholder to seek for another insurance company that may offer a more competitive premium thereby possibly avoiding bearing a higher premium. In such situations, insurers are faced with the challenges of policyholder retention by keeping premiums and possibly expenses low in the face of competition. For every line of insurance, understanding policyholder behavior is an important aspect in the overall operations of an insurance company (see Campbell et al. (2014)). However, the effect of claims on policyholder behavior is quite unique to property and casualty insurance, especially for automobile insurance. The short term nature of the insurance coverage allows policyholders to easily decide whether to switch to another company.

Some work has been done to address the relationship between price and lapse. For example, Dutang (2012) studied the effect of price changes on the renewal of non-life insurance contracts and pointed out that market proxies are important for lapse rate predictions. Guelman and Guillén (2014) proposed a causal inference framework to measure price elasticity in the context of auto insurance and found that higher premiums lead to higher lapse rates. Guelman et al. (2014) pointed out that many insurers would reduce their profits a little in order to increase their renewal rates. Bolancé et al. (2018) investigated optimal prices for customers by assuming that prices have an impact on the probability of renewal.

The main focus of the aforementioned work is on profit maximization. The pre-existing notion of the relationship between policyholder claims and lapse has never been empirically investigated in the actuarial or insurance literature. There is a clear apparent reason for this. It is relatively challenging for insurance companies and researchers to explore this notion because such an analysis requires a follow-up of policyholders switching between companies and capturing the implications of this for analyzing behavioral pattern. Our Singapore dataset is unique and quite suitable for this type of analysis because the dataset contains detailed, micro-level automobile insurance records of all insurance companies in Singapore. It consists of records in three separate files over a nine-year period, covering years 1993-2001, of 45 insurance companies that sell automobile insurance coverage in the country. The policy file has over five million records of policy information, such as type of coverage, vehicle type, driver's age and gender, for each registered car insured in each calendar year. The claims file has under a million records of claims that include dates and amounts of claims filed. The payment file has over four million records of dates, amounts and other useful information about payments made for claims that were filed and recorded in the claims file. Extracts of claims experience of different companies from this same dataset have been used for empirical investigation in Frees and Valdez (2008) and Frees et al. (2009).

In making this dataset useful for our purposes, we have extracted all the claims information, together with policyholder and vehicle characteristics, so that we are able to track the policy switch between calendar years. As part of the preparation of this dataset, the switch has been determined according to the identification of the vehicle information since contract identification cannot be unique among the insurance companies. Based on the dataset used in this paper, we have a total of 893,009 observations of which 324,182 have an indicated policy switch. A policy switch is a binary variable derived from the dataset that indicates an evidence that a policyholder has just changed to a different insurance company. We have removed the observations that did not provide us accurate evidence of switch. For example, there were vehicles for which we may have lost trace possibly because these were sold so that someone else became a new owner of the vehicle with a new vehicle identification.

Now for uncovering interesting relationships between insurance claims and policy switch, we employ a data mining methodology called association rule learning. This technique has its origins 
in the retail industry where a huge amount of data on customer purchases were analyzed to understand consumer buying behavior (see Agrawal et al. (1993)). This data analysis can come in the form of an association rule about relationships of the items purchased. To illustrate, the rule \{ground beef, bun $\} \Rightarrow$ tomato may be drawn from the dataset to suggest that there is a strong likelihood of purchasing tomatoes when ground beef and bun are purchased together. Such mining of information can provide valuable insights to businesses for further promotions and sales, for improving customer relations, and for better management of its product inventories.

Despite its conceptual simplicity, association rule learning has potential applications for a more effective data-driven decision making in a wide variety of disciplines including medical diagnosis, credit card fraud detection and health informatics (see Rajak and Gupta (2008) and Altaf et al. (2017)). Using association analysis, a physician may find association of symptoms for more accurate diagnosis of illness for better patient care. On a similar note, Kost et al. (2012) used this method to derive associations among diseases so that they can compare co-occurrences of diseases at the different levels. Using the data from the 2009 Vernon Uniform Hospital Discharge Data Set with the ICD-9-CM codes to classify diagnoses, the authors were able to identify associations overlapping and new associations among diseases.

In Wong et al. (2005), association rule learning was used and applied in order to achieve optimal direct marketing. It is very important to choose appropriate customers for sending marketing mails because sending mail requires costs. In their paper, they used modified association rule learning and achieved 3.3 times of the profit per mail relative to that of naive method.

It is possible to find an application of association rule learning in actuarial science as well. Lau and Tripathi (2011) used the technique to derive associations between the characteristics of workers and claim types in worker's compensation insurance. They conducted association rule learning on the historical claim data of a waste management company and they find some significant rules, such as $\{$ Day Shift, Foreign Body $\} \Rightarrow\{$ Eye(s) $\}$ and $\{$ Driver, Day Shift, Lowerleg(s) $\} \Rightarrow\{$ Fracture $\}$. By having an understanding of the pattern of the event leading to injuries, the company and the insurers may help determine changes in safety processes in order to prevent future injuries and thereby provide economic incentives.

Association rule learning originated from the work of Agrawal et al. (1993). It is the objective of this technique to mine a big dataset and to draw a connection of the values of the variables in the dataset. Such connection is expressed as an implication of the form $A \Rightarrow B$ where the left-hand side (lhs) A is called the antecedent while right-hand side (rhs) B is called the consequent. The antecedent is a statement of a premise that the condition stated as a consequent is true. The degree of seriousness of this implication can be measured in several ways as discussed in the body of this paper. The purpose of this is straightforward: to mine our Singapore market insurance data to provide us empirical evidence of the relationships among insurance claims, the premium immediately following a claim, and lapse behavior. Ignoring the policyholder and vehicle characteristics, we controlled for in our analysis, our results provided strong evidence of relationships and we were able to deduce association rules in the form:

$$
\{\text { High claim size, Reduced premium }\} \Rightarrow\{\text { Policy switch }\}
$$

We will briefly mention that other more traditional approaches of supervised learning would have made it difficult, if not impossible, to draw such evidence. In traditional approaches of supervised learning (e.g., linear models, generalized linear models), we already have some ideas about the relationships among the variables so that we can come up with some models. In contrast to these traditional approaches of supervised learning, association rule learning is more suitably used as an exploratory tool as we have done so in this paper. In association rule learning, we aim to find some relationships among the variables so that we can use them to build predictive models in the next step. As a result, we caution the reader and user of our results that association rules are difficult to use as predictive models. 
For the rest of this paper, it has been organized as follows. Section 2 provides an overview about association rule learning. We also define the measures commonly used in association rules. Section 3 provides discussion and summarization of the dataset used in our empirical investigation. Section 4 details the results of our analysis. We conclude in Section 5.

\section{Concepts of Association Rule Learning}

Consider a set $\mathcal{I}$ of $m$ items where $\mathcal{I}=\left\{i_{1}, i_{2}, \ldots, i_{m}\right\}$, and denote the dataset $D$ to be the set ofl $N$ transactions represented as $D=\left\{t_{1}, t_{2}, \ldots, t_{N}\right\}$. The items are sometimes called attributes that are often binary variables but could also be categorical variables. None of the items can be continuous and the practice is to convert continuous into categorical variables for meaningful applications of association rule learning. The transaction $t_{k}$ in the dataset, for $k=1,2, \ldots, N$, is a subset of items from the dataset. An itemset $X$ is a collection of zero or more items and we can call the null (or empty) set to be the itemset with zero items. If the itemset $X$ is a subset of transaction $t_{k}$, then we say that the transaction contains the itemset $X$ (see Bramer (2016) and Weiss et al. (2010)).

Given association rule learning has its origin in marketing, the dataset is usually a market basket data with items referring to goods or products purchased. See, for example, the work of Agrawal et al. (1993). For our purposes of illustration, consider the simplified course enrollment data example tabulated in Table 1.

Table 1. An illustrative dataset of class enrollment.

\begin{tabular}{cccccc}
\hline Student ID & Calculus & Physics & Statistics & Latin & History \\
\hline 1 & 1 & 1 & 1 & 0 & 0 \\
2 & 0 & 1 & 1 & 1 & 1 \\
3 & 0 & 1 & 0 & 0 & 1 \\
4 & 1 & 1 & 1 & 1 & 0 \\
5 & 0 & 0 & 1 & 0 & 1 \\
6 & 1 & 1 & 1 & 1 & 0 \\
7 & 1 & 0 & 1 & 0 & 0 \\
8 & 0 & 1 & 0 & 0 & 1 \\
\hline
\end{tabular}

Each of the eight students in this dataset can enroll in any of the five subjects: Calculus, Physics, Statistics, Latin, and History. The subjects are the items in our dataset, each of which is a binary attribute that indicate enrollment in the subject. A value of 1 indicates enrollment in the corresponding course whereas 0 means no enrollment. The listing of courses for each student are the transactions in our dataset; we therefore have a total of eight transactions with each corresponding to a student. For example, Student ID 3 has the transaction $t_{3}=\{$ Physics, History $\}$ while Student ID 6 has the transaction $t_{6}=\{$ Calculus, Physics, Statistics, Latin $\}$. The itemset $X=\{$ Calculus, Physics $\}$ is a subset of transactions $t_{1}, t_{4}$, and $t_{6}$.

Such a course enrollment dataset can provide meaningful information to a university to understand its enrollment pattern in order to meet enrollment needs. Planning for enrollment needs is critical to a university to optimally allocate scarce resources. One of the decision making process for this purpose is to derive meaningful association rules among the different courses. An association rule is indeed expressed as an implication of the form $X \Rightarrow Y$ for disjoint itemsets $X$ and $Y$, that is, $X \cap Y=\varnothing$. If all $m$ possible items are binary attributes, there would be a total number of $m \cdot 2^{m-1}$ possible association rules. In our enrollment dataset with five courses (or attributes), this leads us to a total of 80 possible association rules. Evaluating the interestingness and strength of each possible association rule can clearly be costly and this cost increases exponentially with the number of attributes present. 
Association rule learning is a data mining method for finding meaningful relations among incidence of events with information extracted from these incidences. For our course enrollment dataset, association rules can help the university draw conclusions from queries that are for example:

1. Search for association rules with "Statistics" as the antecedent. Such rules can help the university assess the impact of deciding to discontinue offering this course.

2. Search for association rules with "Physics" as the consequent. Such rules can help the university plan for courses that will lead to an increased enrollment for this course.

3. Search for association rules with "Statistics" as the antecedent and "Physics" as the consequent. Such rules can help the university plan for subjects in addition to "Statistics" that will help further boost enrollment for "Physics".

4. Search for the most attractive, or best, association rules with "Physics" as the consequent. Best can be measured according to the interestingness or strength of such rules.

\subsection{Common Measures Used}

There are important measures used in association rule learning to assist the decision maker in drawing the strength and interestingness of an association rule.

To begin, we introduce the concept of a support which measures the frequency of an itemset. The support of an itemset $X$ is defined as the proportion of observations which contains $X$ in the whole dataset. Mathematically, we write

$$
\operatorname{supp}(X)=\frac{\left|\left\{t_{k} \mid X \subseteq t_{k}, t_{k} \in D\right\}\right|}{N}
$$

where $|\cdot|$ refers to the number of elements in the set. Using our sample dataset, the support of the itemset $X=\{$ Physics, Statistics $\}$ is $4 / 8=0.5$. Support can be an important measure because infrequent itemsets, those with low support, may be immediately discarded or eliminated in mining for association rules. Those itemsets with large support are more highly desirable.

Confidence is a measure that is based on the notion of a support. For a given rule, say $X \Rightarrow Y$, we define confidence as:

$$
\operatorname{conf}(X \Rightarrow Y)=\frac{\operatorname{supp}(X \cup Y)}{\operatorname{supp}(X)}=\frac{\left|\left\{t_{k} \mid(X \cup Y) \subseteq t_{k}, t_{k} \in D\right\}\right|}{\left|\left\{t_{k} \mid X \subseteq t_{k}, t_{k} \in D\right\}\right|}
$$

For a given rule $(X \Rightarrow Y)$, this reliability measure gives us the proportion of the observations in our dataset with all items from $X$ that have also all items from $Y$. The larger this proportion is, the more confident we are for the itemset $Y$ to be present in our observations that contain itemset $X$. In our sample dataset, for the rule $\{$ Physics, Statistics $\Rightarrow \operatorname{Latin}\}$ we have a confidence of $\operatorname{conf}(X \Rightarrow Y)=$ $\frac{3 / 8}{4 / 8}=0.75$. In words, among those students who enroll in both Physics and Statistics, $75 \%$ of the time they will also enroll in Latin.

There are two additional measures of interestingness of association rules that we would like to use in this paper. We define the metric lift of a rule as follows:

$$
\operatorname{lift}(X \Rightarrow Y)=\frac{\operatorname{conf}(X \Rightarrow Y)}{\operatorname{supp}(Y)}=\frac{\operatorname{supp}(X \cup Y)}{\operatorname{supp}(X) \times \operatorname{supp}(Y)}
$$

It is computed as the ratio of the confidence to the support of the consequent in the rule, but it can also be expressed as the ratio of the support of $X \cup Y$ to the product of the support of $X$ and the support of $Y$. This latter expression provides us an interesting interpretation of the lift: if the events associated with the itemsets $X$ and $Y$ are independent, then there is no possible association rule that can be drawn. In effect, the lift is a measure of the degree to which there is presence of dependence.

A lift of 1 indicates there is independence. A lift $>1$ indicates a strong presence of dependence in which case, the association rule is much more potentially useful. In our sample dataset, for the rule 
$\{$ Physics, Statistics $\Rightarrow$ Latin $\}$, we have a lift of lift $(X \Rightarrow Y)=\frac{0.75}{0.375}=2.00$. In this case, an association rule can be meaningfully drawn from with $X$ as the antecedent and $Y$ as the consequent.

Finally, the conviction of a given rule is defined as

$$
\operatorname{conv}(X \Rightarrow Y)=\frac{1-\operatorname{supp}(Y)}{1-\operatorname{conf}(X \Rightarrow Y)}
$$

Conviction is a metric that is used to measure the strength of the association between $X$ and $Y$ than just completely random. The numerator is the frequency of the occurrence of $X$ in the absence of $Y$ in the case of independence. The denominator is the total frequency of $X$ in the absence of $Y$. Using our sample enrollment dataset, for the rule $\{$ Physics, Statistics $\Rightarrow$ Latin $\}$, we have a conviction of $\operatorname{conv}(X \Rightarrow Y)=\frac{1-0.375}{1-0.75}=2.50$.

By re-writing the conviction formula as

$$
\begin{aligned}
\operatorname{conv}(X \Rightarrow Y) & =\frac{1-\operatorname{supp}(Y)}{1-\operatorname{supp}(X \cup Y) / \operatorname{supp}(X)}=\frac{\operatorname{supp}(X)-\operatorname{supp}(X) \operatorname{supp}(Y)}{\operatorname{supp}(X)-\operatorname{supp}(X \cup Y)} \\
& =\frac{\frac{\operatorname{supp}(X)}{\operatorname{supp}(X \cup Y)}-\frac{\operatorname{supp}(X) \operatorname{supp}(Y)}{\operatorname{supp}(X \cup Y)}}{\frac{\operatorname{supp}(X)}{\operatorname{supp}(X \cup Y)}-1}=\frac{1 / \operatorname{conf}(X \Rightarrow Y)-1 / \operatorname{lift}(X \Rightarrow Y)}{1 / \operatorname{conf}(X \Rightarrow Y)-1},
\end{aligned}
$$

we can show the relationships among conviction, confidence, and the lift as follows:

$$
\operatorname{conv}(X \Rightarrow Y)=\frac{1-\operatorname{conf}(X \Rightarrow Y) / \operatorname{lift}(X \Rightarrow Y)}{1-\operatorname{conf}(X \Rightarrow Y)}
$$

Especially for interpretation purposes, it may be imperative to express these metrics in probabilistic terms. By defining $E_{X}$ and $E_{Y}$ to be the respective events of having itemsets $X$ and $Y$, we have a summary of the equivalence Table 2 .

Table 2. The various metrics in probabilistic terms.

\begin{tabular}{llc}
\hline Metric & Notation & Probabilistic Term \\
\hline support & $\operatorname{supp}(X)$ & $\operatorname{Pr}\left(E_{X}\right)$ \\
\hline confidence & $\operatorname{conf}(X \Rightarrow Y)$ & $\operatorname{Pr}\left(E_{Y} \mid E_{X}\right)$ \\
\hline lift & $\operatorname{lift}(X \Rightarrow Y)$ & $\frac{\operatorname{Pr}\left(E_{Y} \cap E_{X}\right)}{\operatorname{Pr}\left(E_{X}\right) \operatorname{Pr}\left(E_{Y}\right)}$ \\
\hline \multirow{2}{*}{ support } & $\operatorname{conv}(X \Rightarrow Y)$ & $\frac{1-\operatorname{Pr}\left(E_{Y}\right)}{1-\operatorname{Pr}\left(E_{Y} \mid E_{X}\right)}$ \\
\hline
\end{tabular}

The association rule metrics are estimates of the corresponding probabilities in this table. For example, the support of $X$ is an estimate of the probability that an observation in the dataset (or transaction) contains the itemset $X$. In addition, the confidence is an estimate of the probability that an observation contains the itemset $Y$, given it contains $X$.

\subsection{The A-Priori Algorithm}

Association rule data mining techniques involve the process of searching for frequent itemsets in the dataset that satisfy a support threshold and then extracting rules from these frequent itemsets. It can be rephrased as a technique involving two tasks:

- $\quad$ Find all the itemsets $X$ which satisfies $\operatorname{supp}(X) \geq$ minsupp, where minsupp is a minimum level of required support as determined according to the purpose of analysis. 
- Utilizing these frequent itemsets, find all the association rules $X \Rightarrow Y$ which satisfies $\operatorname{conf}(X \Rightarrow$ $Y) \geq$ minconf, where minconf $f$ is a minimum level of required confidence.

For details, see Agrawal et al. (1993). Note that although the common practice is to specify a minimum threshold for confidence, because of the relationship among confidence, lift, and conviction, as shown in the previous subsection, this is equivalent to specifying thresholds of these other measures.

Accomplishing the tasks involved in association rule learning can be rather straightforward by searching our dataset for all itemsets and all possible association rules that meet these thresholds. Even with the aid of fast computing, this brute-force approach of searching all possibilities can lead to infinitely many rules that can be difficult to extract and to draw meaningful deductions. One of the earliest and simplest association rule algorithm, the a-priori approach can provide assistance in this regard with an algorithm that reduces the candidate itemsets for consideration of association rules. This reduction procedure, known as support-pruning, is accomplished by iteratively eliminating itemsets that do not satisfy the pre-specified threshold. For an itemset that is considered frequent, then all of its subsets must also be infrequent. The elimination process, according to this a-priori principle, is therefore exercised by removing infrequent itemsets when the converse of this principle is applied.

To demonstrate the effectiveness of this pruning process, consider a dataset with a list of say 10 items. The initial phase of the process is to consider all 1-itemsets, remove the infrequent itemsets, and then consider all 2-itemsets from the reduced list of possible 1-itemsets. To assess how much reduction is accomplished, let us suppose that we have eliminated five 1-itemsets and considered only therefore remaining five 1-itemsets. Then, in the next step, instead of considering all possible 2-itemsets which is equal to $\left(\begin{array}{c}10 \\ 2\end{array}\right)=45$, we would consider only $\left(\begin{array}{l}5 \\ 2\end{array}\right)=10$ possibilities, eliminating therefore 352 -itemsets. This classical approach is indeed based on an iterative process of finding frequent itemsets starting with finding frequent 1-itemsets and eliminating the infrequent ones, then finding 2-itemsets from the remaining frequent itemsets, and so on. In general, the basket of candidate $k$-itemsets are used to search for $(k+1)$-itemsets that meet the specified support criterion.

Once the support-pruning is done, all applicable association rules are then considered and we eliminate those whose confidence thresholds are not satisfied. The generation rule of an association rule is even further simplified if the decision maker can be more specific about its consequent.

For simplicity, especially in terms of interpretation, the a-priori algorithm has been exclusively used in this paper for establishing association rules. For detailed explanation about this and other algorithms used in association rule learning, please see Tan et al. (2006) or Aggarwal (2015).

\section{Data Characteristics}

The aim of this paper is to find empirical evidence about policyholder lapse behavior in the wake of an insurance claim. For a meaningful analysis, we needed not only the claims information from an insurance company and whether the policyholder lapsed subsequent to a claim, but also the additional premium information that can be obtained when the policyholder lapsed.

We based our analysis on a very unique dataset that contains detailed, micro-level automobile insurance records of all insurance companies in Singapore over a nine-year period covering years 1993-2001. Extracts of claims experience of some companies from this same dataset have been used for empirical investigation in Frees and Valdez (2008) and Frees et al. (2009). Despite its size, Singapore has over half a million vehicles on the road today, (https:// data.gov.sg/) and automobile insurance is one of the most important lines of insurance offered by general insurers in the Singapore insurance market. Annual gross premium from this line of insurance has historically been accounted for over a third of the entire insurance market. Just as like in many other developed countries, auto insurance provides coverage at different layers, with the minimum layer that is mandatory, providing protection against death of bodily harm to third parties, regardless of who is at fault. This is called third party liability coverage for many countries such as the United States.

Processing these millions of records in order to extract the meaningful information needed for our purpose has presented us some challenges. First, we have records of 45 different companies during the 
nine-year period, and for each company, we have detailed information about each recorded policy, its history of claims submission and subsequent payments. Second, we needed some information between companies that matches the policyholder and the vehicle insured. In order to track whether a policyholder switch or not, we were able to successfully match the vehicle information between insurance companies. We followed records across calendar years and assigned a policy switch variable which is defined to be a binary variable indicating whether the policyholder of the same insured vehicle switched (Yes $=1$, No $=0$ ) or not. Finally, we removed the observations that did not provide us accurate evidence of switch. For example, there were vehicles for which we may have lost trace possibly because these were sold so that someone else became a new owner of the vehicle with a new vehicle identification. In Singapore, it is also not uncommon to keep cars for only up to 10 years because in an effort to significantly reduce the number of old cars, the government has a program in place that provides incentives to deregister cars before they turn 10 years old.

Our final dataset has a total of 893,009 observations of which $36.3 \%$ have a policy switch of 1 . Figure 1 provides a graphical representation of the relative frequency of itemsets in our dataset.

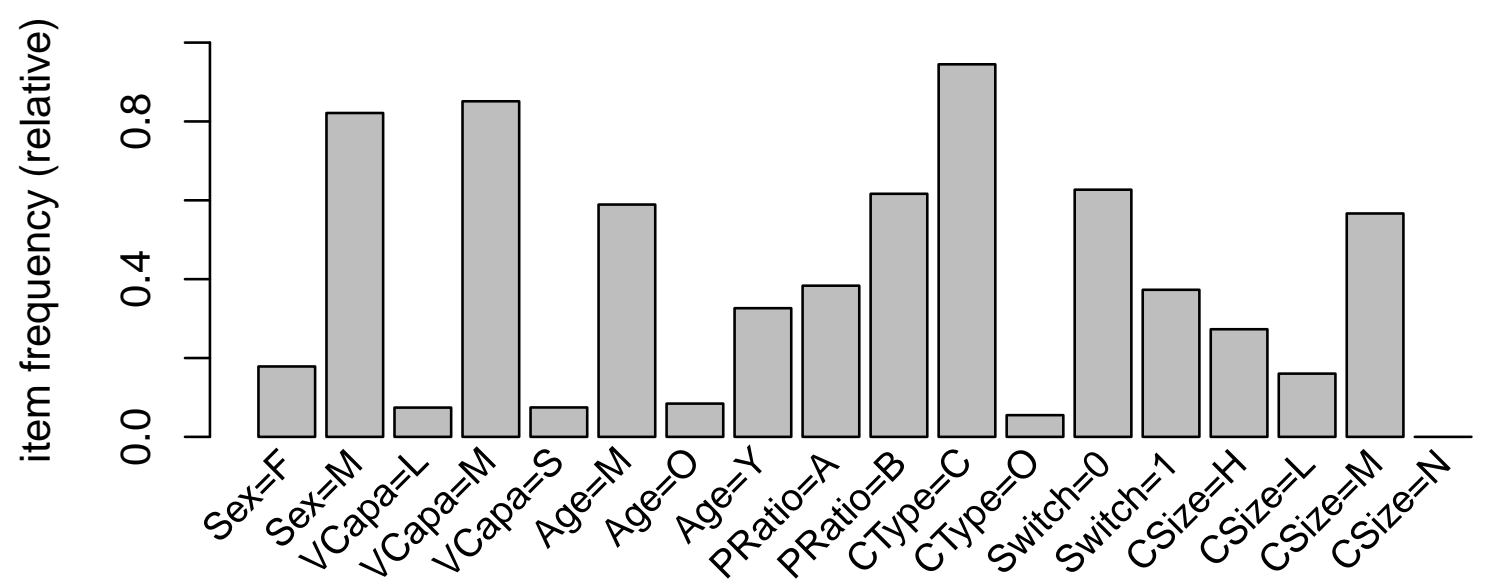

Figure 1. Relative frequency of itemsets in the original dataset.

Table 3 provides a list and description of the nine variables we used together with some simple summary statistics. The observations in our dataset consist of policies with comprehensive coverages for first party property damage and bodily injury as well as third party liability for property damage and bodily injury. The type of insurance coverage is predominantly Comprehensive with $82.9 \%$ of the total observations. The only vehicle characteristic that we can draw from our files is size or vehicle capacity, VCapa, defined to be the engine capacity measured in cubic centimeters (cc). We categorize the vehicles according to three categories of vehicle capacity: (Small, Medium, and Large). Most vehicles in our dataset are classified Medium size capacity, with about $82.5 \%$ of the total observations. We have gender and age that relate to driver information. More than $80 \%$ of the insured drivers are male and about two-thirds are in the middle age range. It is worth noting that due to gender equality issues, gender information is not allowed to be used in ratemaking according to European Union (EU) directives. Since our dataset was obtained from Singapore, we still keep the gender information in our analysis. Furthermore, some jurisdictions use gender for risk classification.

We categorize age according to whether Young (less than 35 years old), Middle (between 35 and 55 years old), and Old (55 years and older). It is worth noting that this categorization makes sense in Singapore. First, there is a very comprehensive public transportation system in Singapore so that driving at early age is not highly encouraged because of this convenience. Second, owning a vehicle in Singapore can be quite expensive and this is because of its size, the government controls the number of vehicles by imposing a large amount tax at purchase and for continued ownership. Finally, especially during the period of our observations, retirement was at about 55 years old, although it is likely that average retirement age today may have gone higher than this age. 
Table 3. Description of variables and summary statistics.

\begin{tabular}{|c|c|c|c|}
\hline Variables & Description & & Proportions \\
\hline \multirow{2}{*}{ Switch } & \multirow{2}{*}{ Indicator for policyholder switch } & Yes $=1$ & $36.3 \%$ \\
\hline & & $\mathrm{No}=0$ & $63.7 \%$ \\
\hline \multirow{2}{*}{ CType } & \multirow{2}{*}{ Type of coverage: } & Comprehensive $=\mathrm{C}$ & $82.9 \%$ \\
\hline & & Others $=\mathrm{O}$ & $17.1 \%$ \\
\hline \multirow{3}{*}{ VCapa } & \multirow{3}{*}{ Capacity of the vehicle: } & Small $(\leq 1000)$ & $9.6 \%$ \\
\hline & & Medium $(\in(1000,2000])$ & $82.5 \%$ \\
\hline & & Large $(>2000)$ & $7.8 \%$ \\
\hline \multirow{2}{*}{ Sex } & \multirow{2}{*}{ Insured's sex: } & Male $=\mathrm{M}$ & $81.6 \%$ \\
\hline & & Female $=\mathrm{F}$ & $18.4 \%$ \\
\hline \multirow{3}{*}{ Age } & \multirow{3}{*}{ Insured's age: } & Young $(<35)$ & $26.7 \%$ \\
\hline & & Middle $(\in[35,55))$ & $62.5 \%$ \\
\hline & & Old $(\geq 55)$ & $10.8 \%$ \\
\hline \multirow{2}{*}{ Claim } & \multirow{2}{*}{ Whether claim is present or not } & Yes $=Y$ & $11.8 \%$ \\
\hline & & $\mathrm{No}=\mathrm{N}$ & $88.2 \%$ \\
\hline \multirow{4}{*}{ ClaimSize } & \multirow{4}{*}{ Amount of claim relative to average } & High $(>3 Q)$ & $3.2 \%$ \\
\hline & & Medium (in $[1 \mathrm{Q}, 3 \mathrm{Q}])$ & $6.7 \%$ \\
\hline & & Low $(<1 \mathrm{Q})$ & $1.9 \%$ \\
\hline & & Without Claim & $88.2 \%$ \\
\hline \multirow{2}{*}{ PremRatio } & \multirow{2}{*}{ Ratio of the premium of this to previous year } & AboveAvg $(>1.14)$ & $22.4 \%$ \\
\hline & & BelowAvg $(\leq 1.14)$ & $77.6 \%$ \\
\hline
\end{tabular}

The probability of having a claim is just as about what we expected: $11.8 \%$ of the observations had at least one claim during a calendar year. Of these observations with at least one claim, one-fifth had claim size below its first quartile (Low), two-thirds had claim size between the first and third quartile (Medium), and the rest had claim size above the third quartile (High). In order to relate premiums to claims and policy switch, we defined a variable called PremRatio which is equal to the ratio of the premium of this calendar year to that of the previous year. A PremRatio larger than one indicates in increase in premium while smaller than one indicates a decrease. We did some preliminary investigation as to what the suitable cutoff is for a premium ratio. Considering only those policies with claims, we find that the average rate of premium increase is $15 \%$ so that we considered any increase above this average to be AboveAvg and below this average to be BelowAvg. For any increase at exactly at $15 \%$, this was considered BelowAvg. Of our total observations, $22.4 \%$ had premium increases that were above average. The choice of the premium rate increase was a reasonable one since we considered only those policies for which there was at least one claim. For many insurance companies, premium rate increase is not uncommon with or without a claim.

Figure 2 provides respective histograms of the premium ratio and the logarithm of the size of the claim, given the policy had a claim. The premium ratio variable has a minimum of 0.01 and a maximum of 4.99 , with average of 1.17 and standard deviation of 0.6 . Although we observe premium ratios as little as 0.01 and as large as 4.99 , such extremes were not frequent in our dataset. Given the policy had a claim size, the size of the claim has a minimum of 0.01 and a maximum of 1,313,613 with average of and standard deviation of 11,179.56. Table 4 provides additional statistics for these two variables describing the premium ratio and the size of the claim (in dollars). It is worth noting at this point that any reference to amounts here are in Singapore dollars.

As we are simply interested in whether a policyholder switch is impacted by the size of the claim and the subsequent change in premium, we present Figure 3 that provides the relationship between the size of claim (in logarithm) and the premium ratio according to whether there was a switch or not. According to this graphical evidence, we observe no relationship or pattern that we can observe. Traditional methods of supervised learning (e.g., generalized linear models) are less suitable in this 
regard. This is one of our motivation for using association rules to seek for evidence of policyholder lapse behavior according to the presence of a claim and the change in premium.

\section{Premium Ratio}

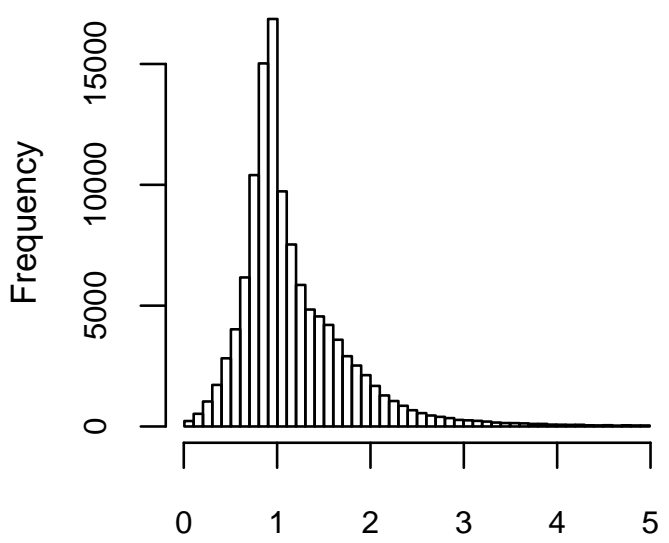

Log of Claim Size

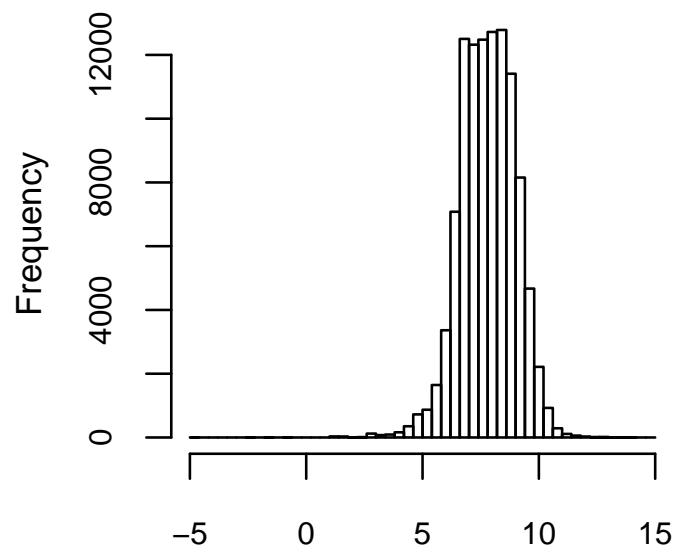

Figure 2. Histograms of premium ratio and log of claim size.
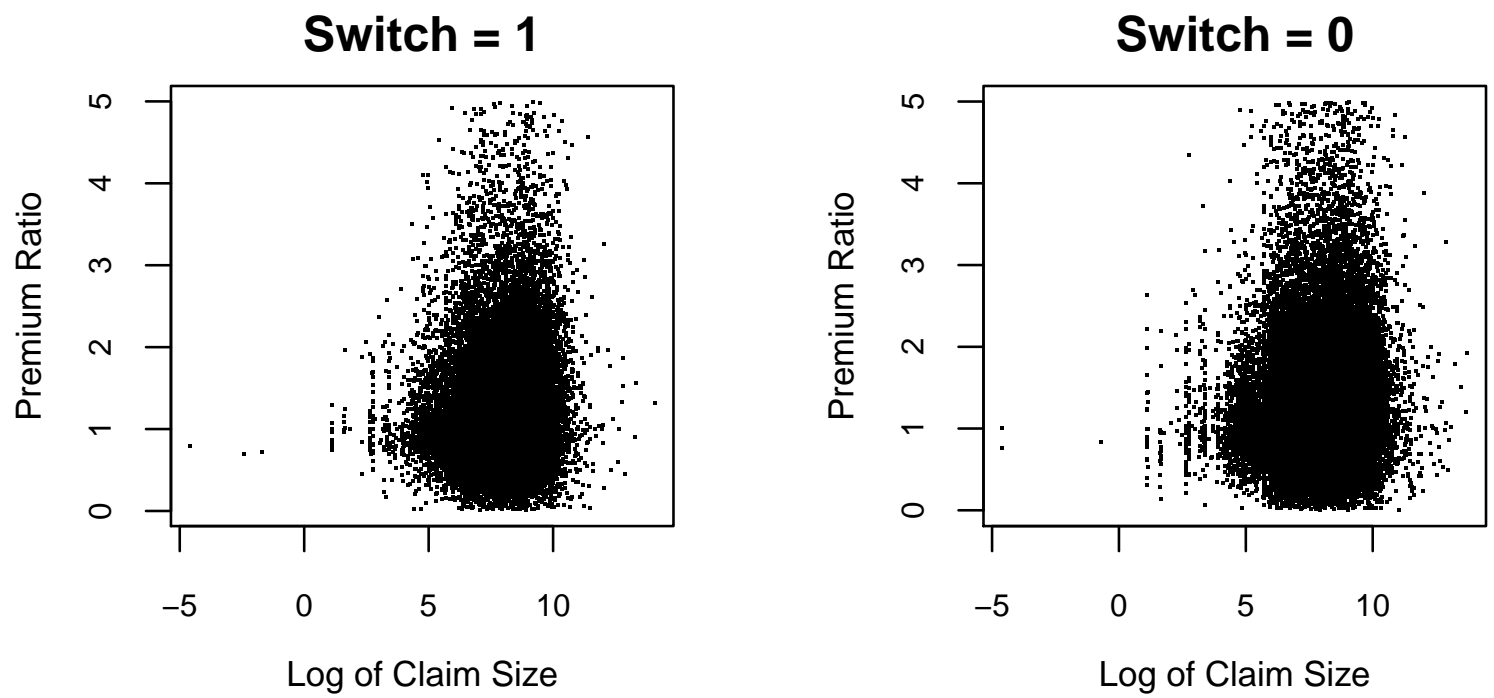

Figure 3. Relationship between log of claim size and premium ratio according to Switch.

Table 4. Summary statistics of premium ratio and claim size

\begin{tabular}{cccccccc}
\hline Variables & Minimum & 1st Q & Median & Mean & 3rd Q & Maximum & Std Deviation \\
\hline Premium ratio & 0.006 & 0.82 & 1.001 & 1.174 & 1.411 & 4.991 & 0.596 \\
Claim size & 0 & 1067 & 2500 & 4788 & 5708 & $1,313,613$ & 11,180 \\
\hline
\end{tabular}

\section{Results of Generating Association Rules}

This section provides details of the results of our analysis of policyholder lapse behavior using the technique of association rule learning. We hypothesize that:

- A policyholder is more likely to switch companies immediately after a claim than not. We feel that the size of the claim has an impact in this regard.

- Given that a claim has occurred and that the size of claim is large enough to warrant a premium rate increase, a policyholder is more likely to switch than not for a moderate level of premium increase. 
Given claim size and premium ratio have potential impact on policyholder lapse behavior, we wanted to employ an interim analysis of generating association rules with each of these variables as a consequent. We use policy characteristics that include type of insurance coverage, vehicle capacity, gender, and age as antecedents. The analysis can be visualized in Figure 4.

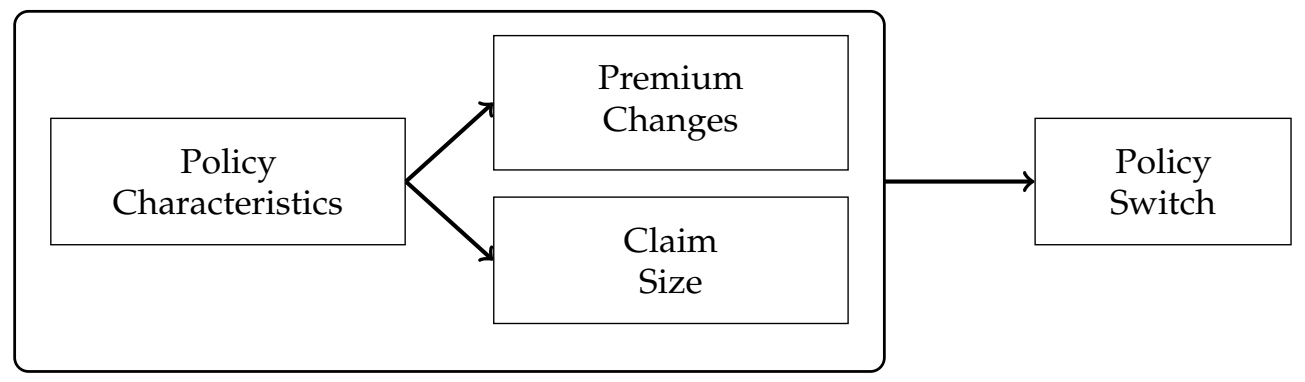

Figure 4. Illustration of decision making flows for each policyholder.

\subsection{Generating Association Rules for Claim Size}

With claim size as the consequent, our algorithm generated three rules summarized in Table 5.

1. A large vehicle capacity and a comprehensive coverage implies a large claim size. This association rule has a support of 0.02 , a confidence of 0.31 , and a lift of 1.13 . Unlike the other two rules below, this rule is independent of insured's sex. This rule gave the highest lift among the three rules.

2. A male driver with a medium vehicle capacity and a non-comprehensive coverage implies a medium claim size. A medium vehicle capacity is generally less expensive and a non-comprehensive coverage leads to payments generally lower than a comprehensive coverage. This association rule has a support of 0.02 , a confidence of 0.61 , and a lift of 1.07 . This rule gave slightly the highest confidence.

3. A male, middle-aged driver with a large vehicle capacity and a comprehensive coverage implies a medium claim size. This rule looks at first glance counterintuitive to the second rule and even the first rule, however, this rule considers the age of the driver. This association rule has a support of 0.03 , a confidence of 0.60 , and a lift of 1.06 . This rule gave slightly the largest support.

A few further comments are necessary about these resulting association rules. First, observe that all three rules led to very small percentage of support. Our dataset is quite large, so this is not a major concern. Second, all three rules do not also lead to very high confidence and very high lift. The lift close to 1 indicates independence between the antecedent and the consequent so that these rules are not quite meaningful. This analysis did not generate meaningful association rules and according to us, association rule is not the correct method to mine this data for understanding the size of the claim. While this is meaningful for exploratory analysis, traditional methods of supervised learning such as regression analysis and generalized linear models may be more suitable.

Table 5. Association rules for claim size based on policy characteristics.

\begin{tabular}{lcccccc}
\hline lhs & rhs & supp & conf & lift & conv & count \\
\hline VCapa $=\mathrm{L}$, CType $=$ C & CSize $=\mathrm{H}$ & 0.02 & 0.31 & 1.13 & 1.05 & 2303 \\
Sex $=$ M, VCapa $=$ M, CType $=$ O & CSize $=$ M & 0.02 & 0.61 & 1.07 & 1.10 & 2367 \\
Sex = M, VCapa $=$ L, Age = M, CType $=$ C & CSize $=$ M & 0.03 & 0.60 & 1.06 & 1.08 & 2880 \\
\hline
\end{tabular}

Figure 5 is an interesting graph that provides the connection of the items in the rules for claim size. This figure provides a visualization of the three association rules summarized in Table 5 which each circle corresponding to an association rule. In the upper portion of the graph, we see the items that directly impact high claim size and we also observe that this provides the largest lift. In the middle 
portion of the graph, we see several items that directly impact medium claim size. The largest circle in the middle indicates high support.

\section{Visualizing the connection of the items in the rules for claim size}

size: support $(0.022-0.027)$

color: lift $(1.057-1.131)$

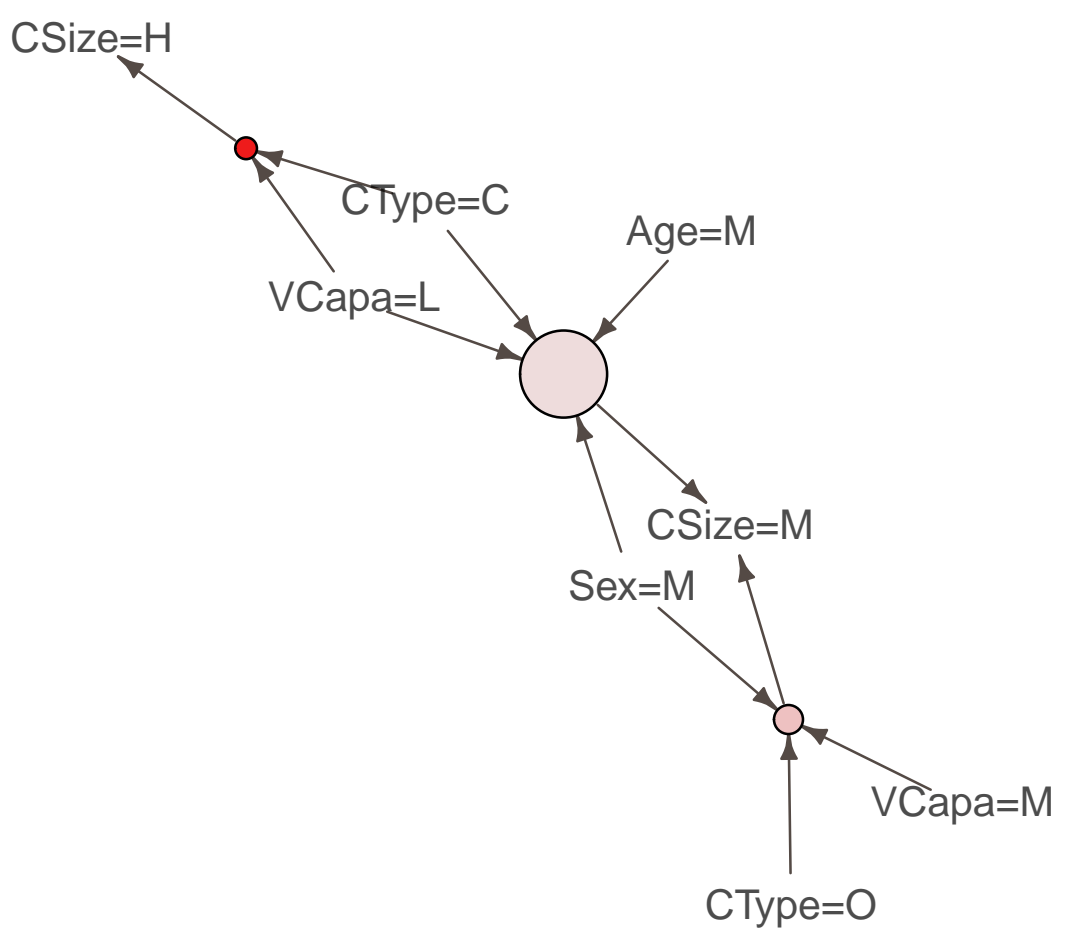

Figure 5. Graph of association rules for claim size.

\subsection{Generating Association Rules for Premium Ratio} Table 6 .

With premium ratio as the consequent, our algorithm generated three rules summarized in

1. A male driver of a medium vehicle capacity with a non-comprehensive coverage implies an above average premium ratio. This association rule has a support of 0.02 , a confidence of 0.60 , and a lift of 1.56. This rule is independent of driver's age and it gives the better confidence and the better lift among the three rules.

2. An old driver of a medium vehicle capacity with a comprehensive coverage also implies an above average premium ratio. This association rule has a support of 0.02 , a confidence of 0.42 , and a lift of 1.11. This rule has the worst confidence among the three rules.

3. A young driver of a small vehicle capacity with a comprehensive coverage implies a below average premium ratio. This association rule has a support of 0.02 , a confidence of 0.68 , and a lift of 1.10. This rule has the highest confidence but slightly the worst lift.

The first of these rules provides for a more meaningful association rule with a decent confidence and a lift much larger than 1 . The connection of the items in the association rules for premium ratio can be visualized in Figure 6. Here we note that at the middle portion of the figure, there are more items that directly impact the premium ratio than either at the top or bottom portion. 


\section{Visualizing the connection of the items in the rules for premium ratio size: support $(0.022-0.023)$ color: lift $(1.104-1.56)$}

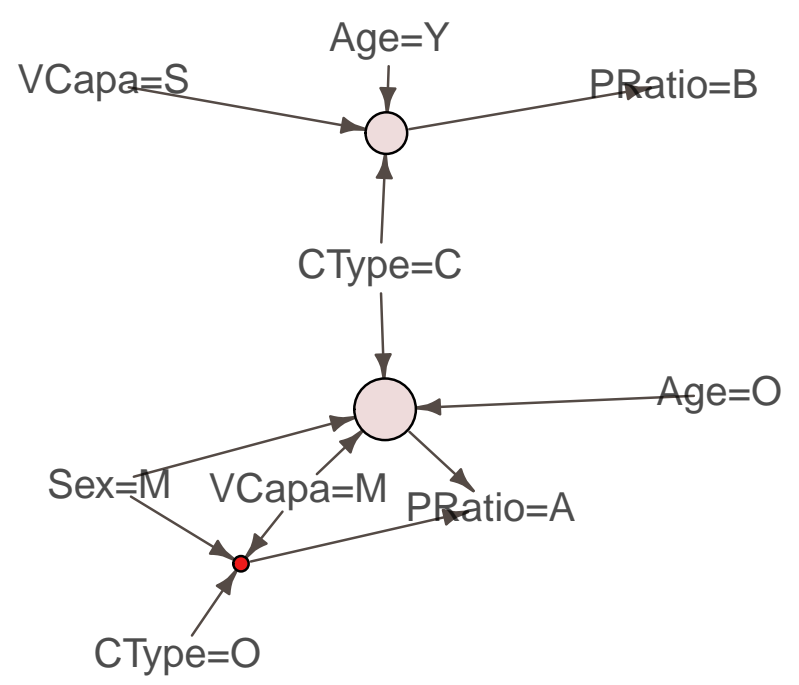

Figure 6. Graph of association rules for premium ratio.

Table 6. Association rules for premium ratio based on policy characteristics.

\begin{tabular}{lcccccc}
\hline lhs & rhs & supp & conf & lift & conv & count \\
\hline Sex $=$ M, VCapa $=$ M, CType = O & PRatio = A & 0.02 & 0.60 & 1.56 & 1.53 & 2334 \\
Sex $=$ M, VCapa $=$ M, Age = O, CType $=$ C & PRatio = A & 0.02 & 0.42 & 1.11 & 1.07 & 2438 \\
VCapa $=$ S, Age $=$ Y, CType = C & PRatio = B & 0.02 & 0.68 & 1.10 & 1.20 & 2393 \\
\hline
\end{tabular}

\subsection{Generating Association Rules for Policy Switch}

Finally, we put together the effect of policy characteristics, size of claim, and premium ratio to understand their implications on policyholder lapse behavior. With policy switch as the primary consequent, our algorithm generated 10 rules summarized in Table 7 . In particular, we generated rules with implications that a policyholder exercised a policy switch. As stated in the introduction, broadly speaking, these association rules come in the form:

\section{$\{$ High claim size, Reduced premium $\} \Rightarrow\{$ Policy switch $\}$}

This is the very empirical evidence that strongly supports our stated hypotheses. For a policyholder with a large claim size, it is likely that this will lead to an increase in premium if the policyholder remains with the same insurer. On the other, this is a motivation for this same policyholder to seek for an insurer that may provide him for a coverage at a lower premium.

We describe these association rules in more details below in the same order or rules as listed in Table 7. This lists consider policy characteristics apart from claim size and premium ratio. All conviction measures for all association rules generated indicate a high percentage of accuracy as compared to purely random. For example, the first association rule provides a conviction of 1.42 which means that there we are $42 \%$ correct that the association holds than just purely random.

1. A male, young driver with a large claim size and below average premium ratio implies a policy switch. This association rule has a support of 0.02 , a confidence of 0.56 , and a lift of 1.50. This rule is one of the three association rules that produced the highest confidence and largest lift. 
2. A male, young driver of a medium vehicle capacity with a large claim size and below average premium ratio implies a policy switch. This association rule has a support of 0.02 , a confidence of 0.56 , and a lift of 1.50 . This rule is also one of the three association rules that produced the highest confidence and largest lift. When compared to the previous rule, the additional information about driving a car with a medium vehicle capacity also does not affect the metrics resulting from the association rule.

3. A male, young driver with a comprehensive coverage and with a large claim size and below average premium ratio implies a policy switch. This association rule has a support of 0.02 , a confidence of 0.56 , and a lift of 1.50 . This rule is another one of the three association rules that produced the highest confidence and largest lift. When compared to the first rules, the additional information about having a comprehensive coverage does not affect the metrics resulting from the association rule.

4. A young driver with a large claim size and below average premium ratio implies a policy switch. This association rule has a support of 0.02 , a confidence of 0.56 , and a lift of 1.49 . This is very interesting because according to this association rule, a young driver with a high claim and ability to acquire a lower premium has a high motivation to switch policies.

5. A young driver with a comprehensive coverage, a large claim size, and below average premium ratio implies a policy switch. This association rule has a support of 0.03 , a confidence of 0.56 , and a lift of 1.49. The only difference between this rule to that of the third rule is the additional knowledge that the driver is a male. When compared to the previous rule, the additional knowledge of a comprehensive coverage does not generally affect the metrics resulting from the association rule.

6. A young driver of a medium vehicle capacity with a large claim size and below average premium ratio implies a policy switch. This association rule has a support of 0.02 , a confidence of 0.56 , and a lift of 1.49. This rule has vehicle capacity in the antecedent while the previous rule has the type of coverage in the antecedent.

7. A young driver of a medium vehicle capacity with a comprehensive coverage and with a large claim size and below average premium ratio implies a policy switch. This association rule has a support of 0.02 , a confidence of 0.55 , and a lift of 1.49 . The additional knowledge of having a comprehensive coverage generates an association rule almost identical to that of the previous rule.

8. A male, young driver with a comprehensive coverage and with a large claim size implies a policy switch. This association rule has a support of 0.04 , a confidence of 0.50 , and a lift of 1.34 . This is one of the last three rules that do not have the effect of premium ratio.

9. A male, young driver with solely a large claim size implies a policy switch. This association rule has a support of 0.04 , a confidence of 0.50 , and a lift of 1.34 . This is quite similar to the previous rule with the only difference of knowing the driver has a comprehensive coverage.

10. Finally, a male, young driver of a medium vehicle capacity with a comprehensive coverage and a large claim size also implies a policy switch. This association rule has a support of 0.03 , a confidence of 0.50 , and a lift of 1.34 . This is quite similar to the eighth association rule with the only difference of knowing that the insured drives a car with a medium vehicle capacity.

The first seven association rules listed above share similarities including the level of the various metrics used while the last three association rules share other types of similarities with about the same degree of metrics. See Figure 7. Broadly speaking, we can claim that the first seven are slightly more superior than the last three association rules listed above. 
Relationships of the metrics for the rules for policy switch

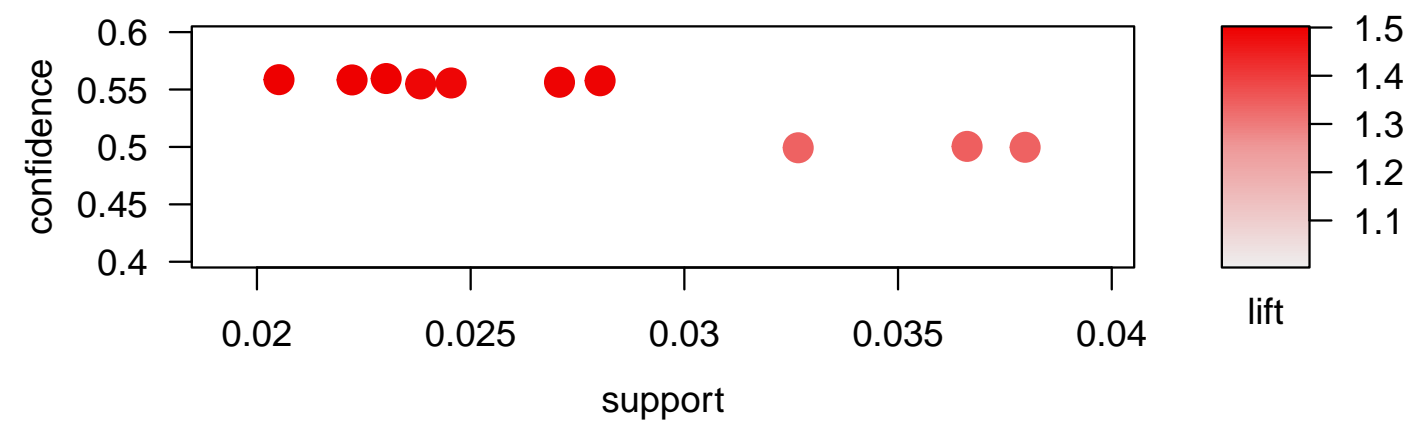

Figure 7. Relationships among support, confidence, and lift.

Figure 8 provides a graphical display of the relative importance of the different items affecting policy switch. Refer to Figure 1 for a comparison of the items were impacted by the association rules. In general, we can draw the following association rules:

$\{$ Young and male driver, High claim size, Reduced premium $\} \Rightarrow\{$ Policy switch $\}$

and

$$
\{\text { Young and male driver, High claim size }\} \Rightarrow\{\text { Policy switch }\}
$$

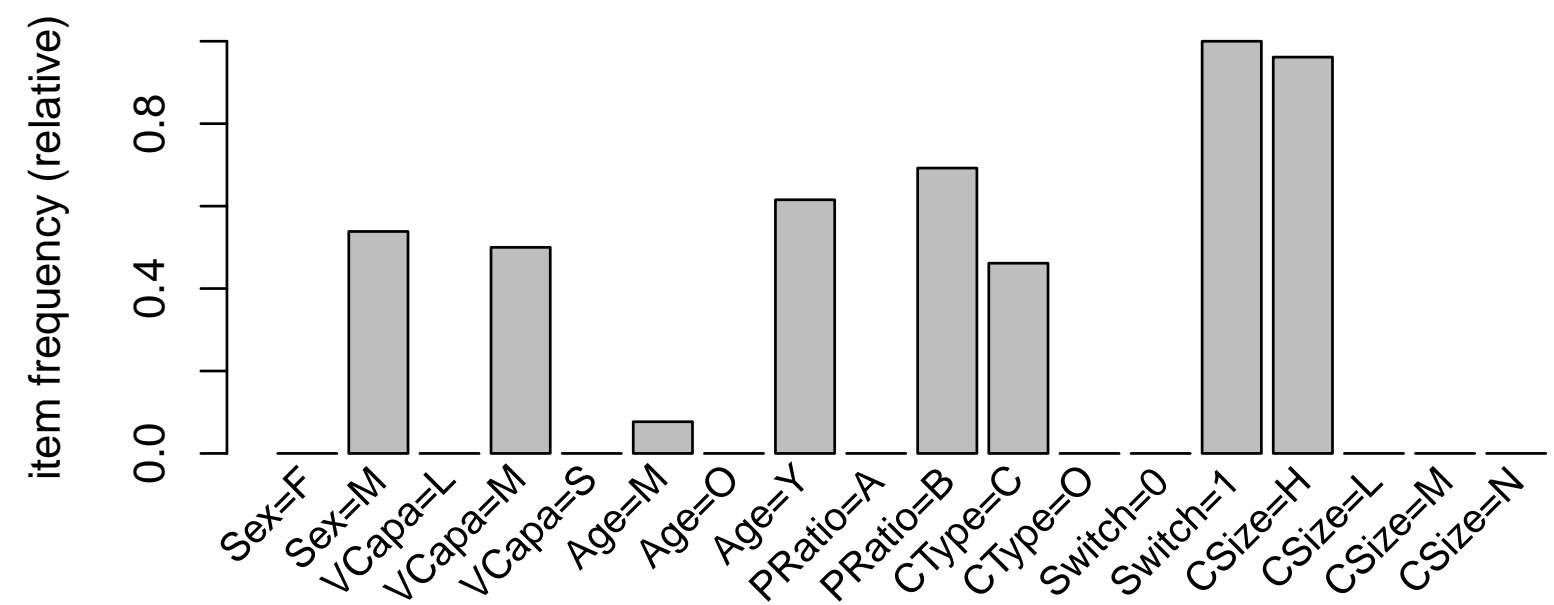

Figure 8. Relative frequency of itemsets in the association rules for policy switch.

Finally, Figure 9 provides a graphical display of the connection of the items in the 10 association rules for policy switch. Each circle represents an association rule, with the big circles indicating large support while the smaller but darker circles indicating higher lift. 
Table 7. Association rules for switch with policy characteristics, claim size, and premium ratio.

\begin{tabular}{|c|c|c|c|c|c|c|}
\hline lhs & rhs & supp & conf & lift & conv & count \\
\hline Sex $=$ & 1 & 0.02 & 0.56 & 1.50 & 1.42 & 2424 \\
\hline Sex $=$ M, VCapa $=$ M, Age $=$ Y, PRatio $=B$, CSize $=H$ & Switch $=1$ & 0.02 & 0.56 & 1.50 & 1.42 & 2160 \\
\hline , Age $=$ Y, PRatio $=$ B, CType $=C$, CSize $=H$ & Switch $=1$ & 0.02 & 0.56 & 1.50 & 1.42 & 2340 \\
\hline Age $=$ Y, PRatio $=\mathrm{B}, \mathrm{CSiz}$ & Switch & 0.03 & 0.56 & 1.49 & 1.42 & 2951 \\
\hline$=\mathrm{C}, \mathrm{CSiz \epsilon}$ & Switcl & 0.03 & 0.56 & 1.49 & 1.41 & 2851 \\
\hline VCapa $=$ M, Age $=$ Y, PRatio $=B$, CSize $=H$ & Switc & 0.02 & 0.56 & 1.49 & 1.41 & 2584 \\
\hline VCapa $=$ M, Age $=$ Y, PRatio $=$ B, CType $=$ C, CSize $=H$ & Switch $=1$ & 0.02 & 0.55 & 1.49 & 1.41 & 2509 \\
\hline $\mathrm{C}, \mathrm{CSize}=\mathrm{H}$ & Switch & 0.04 & 0.50 & 1.34 & 1.26 & 3855 \\
\hline Sex $=M$, Age $=Y$, CSize $=H$ & Switch $=1$ & 0.04 & 0.50 & 1.34 & 1.25 & 3998 \\
\hline Sex $=$ M, VCapa $=$ M, Age $=$ Y, CType $=C$, CSize $=H$ & Switch $=1$ & 0.03 & 0.50 & 1.34 & 1.25 & 3439 \\
\hline
\end{tabular}

\section{Visualizing the connection of the items in the rules for policy switch}

size: support $(0.021-0.038)$
color: lift $(1.338-1.5)$

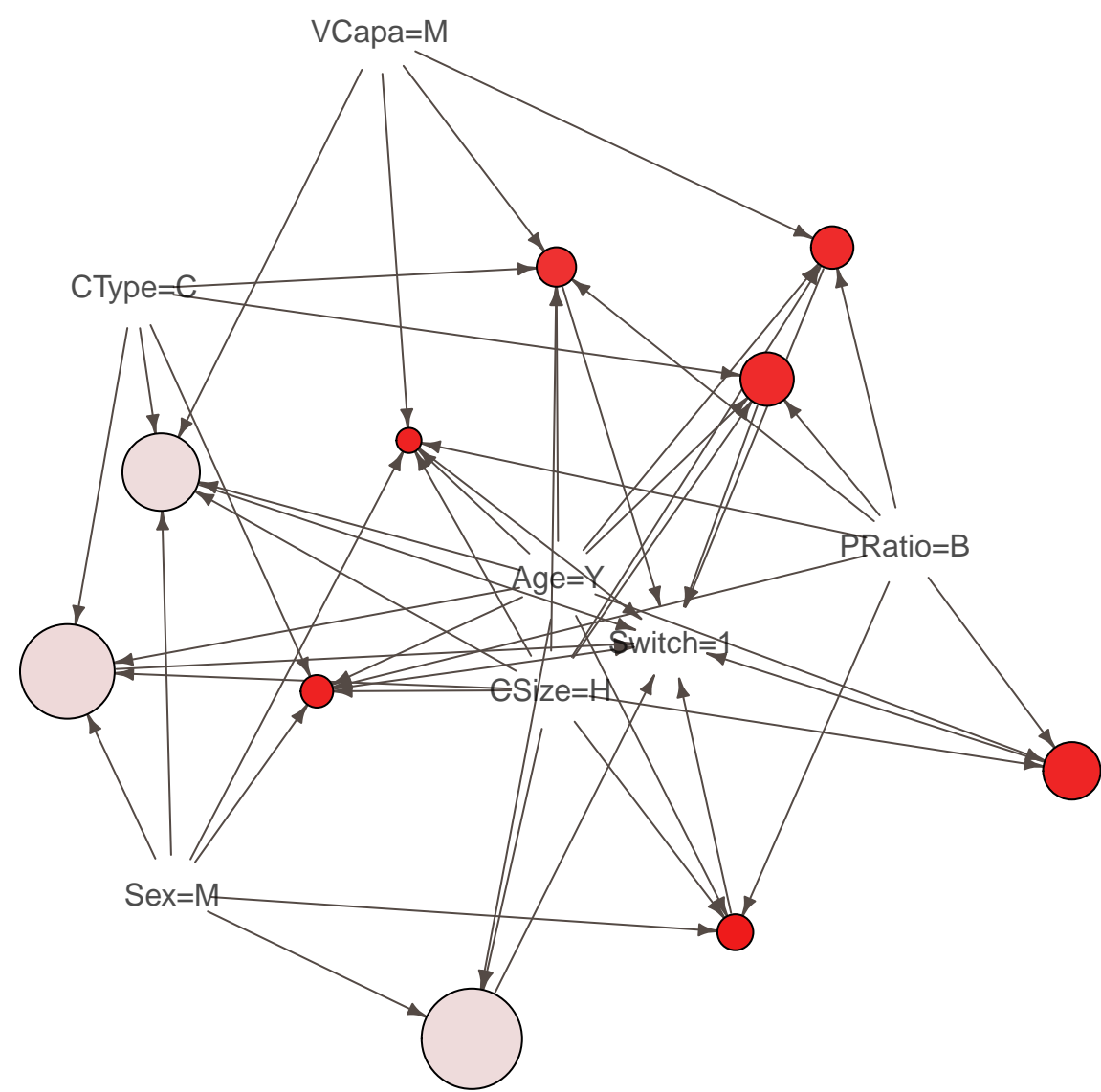

Figure 9. Graph of association rules for policy switch.

\section{Concluding Remarks}

In the insurance industry, there is increased interest in developing strategies for retaining policyholders even after a claim with little sacrifice for a lower profit margin. Policyholder retention 
have a positive effect on the company's reputation for building good customer relationships which could further help attract new customers. It is well known that when experience rating is employed, a policyholder with at least one claim during a policy year will likely have an increase in premium in the subsequent policy year. Such is the core feature of an insurance market based on a bonus-malus system, that is, a policyholder is penalized after a claim. Depending on the level of premium increase, the policyholder may feel more likely to seek for another insurer willing to provide for a cheaper coverage. Sometimes, for example in the case of automobile insurance, a claim may change the driving behavior of the insured. Feeling the pressure of a further increase in premium, the driver may be more careful so that there is strong possibility of a better risk to the insurer. Insurers are therefore faced with the challenges of keeping premiums low, without too much sacrifice of profit margins, in order to retain policyholder loyalty. There has been no prior studies that provide for an empirical evidence of the possible association between policyholder switching after a claim and the associated change in premium. Using the method of association rule learning, a data mining technique that originated in marketing for analyzing and understanding consumer purchase behavior, we are able to provide evidence of such association in this article. This empirical investigation was made possible because of the unique dataset we have. We used a nine-year claims data for the entire Singapore automobile insurance market that allowed us to track information before and after a policy switch. Our results provide evidence of a strong association among the size of the claim, the level of premium increase, and policy switch. We attribute this to the possible inefficiency of the insurance market because of the lack of sharing and exchange of claims history among the companies. As possible future work, we would like to build predictive models to investigate the financial implications of such associations.

Author Contributions: While both G.G. and E.A.V. conceived the primary idea of the methodology used, H.J. joined in the discussion to further explore the literature. In consultation with Jeong and Valdez, G.G. wrote the code to prepare the data for analysis. In consultation with G.G. and E.A.V., H.J. analyzed the data and produced the output for analysis. All three authors had periodic meetings to discuss the literature, the results of the data analysis, and the writing of the manuscript.

Funding: This research has received funding from the Society of Actuaries through our Center of Actuarial Excellence (CAE) research grant on data mining.

Conflicts of Interest: The authors declare no conflict of interest.

\section{References}

Aggarwal, Charu C. 2015. Data Mining: The Textbook. Berlin: Springer.

Agrawal, Rakesh, Tomasz Imieliński, and Arun Swami. 1993. Mining association rules between sets of items in large databases. Paper presented at the 1993 ACM SIGMOD International Conference on Management of Data, Washington, DC, USA, May 25-28, vol. 22, pp. 207-16.

Altaf, Wasif, Muhammad Shahbaz, and Aziz Guergachi. 2017. Applications of association rule mining in health informatics: A survey. Artificial Intelligence Review 47: 313-40. [CrossRef]

Bolancé, Catalina, Montserrat Guillén, Jens Perch Nielsen, and Fredrik Thuring. 2018. Price and profit optimization for financial services. Risks 6: 9. [CrossRef]

Bramer, Max. 2016. Principles of Data Mining. Berlin: Springer.

Campbell, Jason, Michael Chan, Kate Li, Louis Lombardi, Lucian Lombardi, Marianne Purushotham, and Anand Rao. 2014. Modeling of Policyholder Behavior for Life Insurance and Annuity Products: A Survey and Literature Review. Schaumburg: Society of Actuaries.

Dutang, Christophe. 2012. The Customer, the Insurer and the Market. Bulletin Français d'Actuariat. Paris: Institutdes Actuaires.

Frees, Edward W., Peng Shi, and Emiliano A. Valdez. 2009. Actuarial applications of a hierarchical insurance claims model. ASTIN Bulletin 39: 165-97. [CrossRef]

Frees, Edward W., and Emiliano A. Valdez. 2008. Hierarchical insurance claims modeling. Journal of the American Statistical Association 103: 1457-69. [CrossRef]

Guelman, Leo, and Montserrat Guillén. 2014. A causal inference approach to measure price elasticity in automobile insurance. Expert Systems with Applications 41: 387-96. [CrossRef] 
Guelman, Leo, Montserrat Guillén, and Ana M. Pérez-Marin. 2014. A survey of personalized treatment models for pricing strategies in insurance. Insurance: Mathematics and Economics 58: 68-76. [CrossRef]

Kost, Rhonda, Benjamin Littenberg, and Elizabeth S Chen. 2012. Exploring generalized association rule mining for disease co-occurrences. Paper presented at AMIA Annual Symposium Proceedings, Chicago, IL, USA, November 3-7, vol. 2012, p. 1284. Bethesda: American Medical Informatics Association.

Lau, Lucas and Arun Tripathi. 2011. Mine your business-A novel application of association rules for insurance claims analytics. In CAS E-Forum. Arlington: Casualty Actuarial Society.

Lemaire, Jean. 1985. Automobile Insurance: Actuarial Models. Hingham: Kluwer.

McClenahan, Charles L. 2001. Foundations of Casualty Actuarial Science. Arlington: Casualty Actuarial Society.

Rajak, Akash, and Mahendra K. Gupta. 2008. Association rule mining: Applications in various areas. Paper presented at International Conference on Data Management, Beijing, China, April 27-30, Ghaziabad: Institute of Management Technology.

Tan, Pang-Ning, Michael Steinbach, and Vipin Kumar. 2006. Introduction to Data Mining. Delhi: Pearson Education. Weiss, Sholom M, Nitin Indurkhya, Tong Zhang, and Fred Damerau. 2010. Text Mining: Predictive Methods for Analyzing Unstructured Information. Berlin and Heidelberg: Springer Science \& Business Media.

Wong, Ke Wang, Senqiang Zhou, Qiang Yang, and Jack Man Shun Yeung. 2005. Mining customer value: From association rules to direct marketing. Data Mining and Knowledge Discovery 11: 57-79. [CrossRef]

(C) 2018 by the authors. Licensee MDPI, Basel, Switzerland. This article is an open access article distributed under the terms and conditions of the Creative Commons Attribution (CC BY) license (http://creativecommons.org/licenses/by/4.0/). 